\title{
Operative and clinical outcomes of minimally invasive living-donor surgery on uterus transplantation: a literature review
}

\author{
Yusuke Matoba $^{1}$, Iori Kisu ${ }^{1}$, Kouji Banno ${ }^{1}$, and Daisuke Aoki ${ }^{2}$ \\ ${ }^{1}$ Keio University School of Medicine \\ ${ }^{2}$ Affiliation not available
}

November 13, 2020

\begin{abstract}
The surgical approach and choice of drainage veins for uterus transplantation living-donor surgery have been investigated to reduce invasiveness. Of the 51 operations within 26 articles reviewed, the mean operative time was shortest in the laparoscopic approach, and longest in the robot-assisted approach. The mean blood loss was less in the laparoscopic and robot-assisted approaches than in the open approach. In cases where the uterine veins were not preserved, the mean operative time was shortened by each approach and the mean blood loss decreased with the laparoscopic and robot-assisted approaches. These procedures may contribute to less invasive living-donor surgery.
\end{abstract}

Title : Operative and clinical outcomes of minimally invasive living-donor surgery on uterus transplantation: a literature review

Running title: Minimally invasive UTx donor surgery

Author: Yusuke Matoba

Co-authors: Iori Kisu, Kouji Banno, Daisuke Aoki

Institution:

Department of Obstetrics and Gynecology, Keio University School of Medicine

35 Shinanomachi, Shinjku-Ku, Tokyo, Japan

Corresponding author:

Iori Kisu, M.D., Ph.D.

Department of Obstetrics \& Gynecology

Keio University School of Medicine

35 Shinanomachi, Shinjyuku-ku, Tokyo, 1608582, Japan

Phone: +81-3-3353-1211

E-mail: iori71march@a7.keio.jp

\section{Abstract}

The surgical approach and choice of drainage veins for uterus transplantation living-donor surgery have been investigated to reduce invasiveness. Of the 51 operations within 26 articles reviewed, the mean operative time was shortest in the laparoscopic approach, and longest in the robot-assisted approach. The mean blood 
loss was less in the laparoscopic and robot-assisted approaches than in the open approach. In cases where the uterine veins were not preserved, the mean operative time was shortened by each approach and the mean blood loss decreased with the laparoscopic and robot-assisted approaches. These procedures may contribute to less invasive living-donor surgery.

\section{Keywords}

uterus transplantation, living donor surgery, laparotomy, laparoscopy, robot assisted, uterine vein, ovarian vein, utero-ovarian vein

\section{Introduction}

Absolute uterine factor infertility (AUFI) includes congenital uterine malformation and defects, such as Mayer-Rokitansky-Küster-Hauser (MRKH) syndrome, ${ }^{1}$ which occurs in one in 5000 women; acquired uterine defects caused by treatment of uterine cancers or hysterectomy due to puerperal bleeding; and Asherman's syndrome, in which the endometrium is adhered. ${ }^{2}$

A new transplantation technique, uterine transplantation (UTx), has been clinically applied in recent years for the treatment of AUFI. UTx was first performed in Saudi Arabia in 2000. ${ }^{3}$ Although the world's first UTx failed with the removal of a transplanted uterus, basic research using animal models was continued, and in 2014, a Swedish team reported the first live birth after UTx. ${ }^{4}$ Since then, UTx has been applied clinically in many countries, and there have been some reports of live births from women who have undergone UTx. ${ }^{5}$

However, there are medical, ethical, and social challenges to UTx. One of the medical challenges is the highly invasive procedure for living donors. In UTx living-donor surgery, the uterine artery is usually used for the arterial vessel, but there are several venous options. The uterine vein (UV), a branch of the internal iliac vein, is widely used, as by the Swedish team that obtained the first live birth after UTx. The procedure has become the gold standard for UTx living-donor surgery. ${ }^{6}$ When the UV is used, the surgical operation is similar to radical hysterectomy. As the surgical isolation of the UV is performed in a narrow and deep area of the pelvis and there is a complex network of vessels, the procedure is sometimes difficult, resulting in longer surgical time and massive haemorrhage. In addition, as the procedure is performed near the hypogastric nerve, there is a risk of postoperative complications such as dysuria in the living donor. ${ }^{7}$

To solve this problem, the use of ovarian veins (OV) and utero-ovarian veins (UOV) as drainage veins has been investigated (Fig. 1). ${ }^{8}$ When these veins are used, the surgical technique is easier because the vessels to be preserved are in a more superficial layer than when the UV is preserved. In addition, UTx living-donor surgery was initially performed using an open approach, but recently there have been reports of laparoscopic ${ }^{9}$ and robot-assisted approaches ${ }^{10}$ for donor surgery.

In this review of the literature, we report on the differences in surgical and clinical outcomes by the variation of surgical approach and the preserved veins in UTx living-donor surgery.

\section{Method}

\section{Search strategy}

A thorough search of the PubMed database was conducted. The search was not limited by language or date of publication. The search strategies were as follows: (uterus[Title/Abstract] OR uterine[Title/Abstract] OR womb[Title/Abstract]) AND (transplantation OR transplant) AND ("surgery"[Title/Abstract] OR operation[Title/Abstract] OR laparoscopy[Title/Abstract] OR laparoscopic[Title/Abstract] OR robot[Title/Abstract] OR robotic[Title/Abstract] OR laparotomy[Title/Abstract] OR vein[Title/Abstract] OR veins[Title/Abstract] OR venous[Title/Abstract] OR anastomosis[Title/Abstract] OR ovarian[Title/Abstract] OR uteroovarian[Title/Abstract] OR utero-ovarian[Title/Abstract] OR living[Title/Abstract] OR donor[Title/Abstract] OR livebirth[Title/Abstract] OR live-birth[Title/Abstract] OR human). The data were collected on 13 October, 2020.

Eligibility assessment 
Two reviewers (Y.M. and I.K.) independently assessed each article and determined eligibility for inclusion in the review article. Inclusion criteria were English peer-reviewed articles reporting one of the following: (i) surgical information (operative approach, surgical time, blood loss, types and numbers of veins, and operative complications); or (ii) postoperative course (discharge timing, graft failure, and live birth after UTx). Articles regarding animal research on UTx, UTx on deceased donors, not original articles (video article, review, letter to the editor, commentary, and editorial), not written in English, or that did not report the information above were excluded.

Data extraction and analysis

The included studies were reviewed by two independent reviewers (Y.M. and I.K.), and relevant data were extracted including the number of performed human UTx cases, surgical approach of living-donor surgery (open approach, laparoscopic approach, or robot-assisted approach), surgical time, blood loss during donor surgery, the types and numbers of removed veins (UV, UOV, or OV), operative complications, discharge timing, and live birth after UTx.

The data were classified into open, laparoscopic, and robot-assisted approaches for analysis.

The data were also classified and analysed according to whether the UV was removed within each approach.

\section{Results}

This review included 26 original articles (Fig. 2). Reports of living-donor uterus transplants from Saudi Arabia, ${ }^{3}$ Sweden, ${ }^{4,6,11-19}$ China, ${ }^{10,20}$ USA (Dallas), ${ }^{8,21-24}$ Czech Republic, ${ }^{7,25,26}$ Germany, ${ }^{27,28}$ and India ${ }^{9,29}$ were identified, and 51 living-donor UTx were incorporated. The surgical information and clinical data for each case are shown in Table 1. In one case in Germany, the uterus was removed from a donor, but was found to be unsuitable for transplantation during back table processing, and the transplant was not performed. In another case, the uterine veins were not used for transplantation, even though they were preserved and removed from the donor in Czech case.

Of the 51 living-donor UTx cases, the open approach was used in 33 cases, the laparoscopic approach in four cases, and the robot-assisted approach in 14 cases. The data of each approach are summarised in Table 2. The average operative time was 8 hours 26 minutes \pm 2 hours 47 minutes for the open approach, 3 hours 30 minutes \pm 0 hour 33 minutes for the laparoscopic approach, and 10 hours 59 minutes \pm 1 hours 45 minutes for the robot-assisted approach, with a trend toward shorter operative times for the laparoscopic approach and longer operative times for the robot-assisted approach. The mean blood loss was $715 \pm 584$ $\mathrm{mL}$ with the open approach, $100 \pm 0 \mathrm{ml}$ with the laparoscopic approach, and $209 \pm 182 \mathrm{~mL}$ with the robot-assisted approach, with a trend toward less blood loss with minimally invasive procedures, such as the laparoscopic and robot-assisted approaches. The day of discharge was $6.2 \pm 1.3$ postoperative days on average with the open approach, $6.5 \pm 0.5$ days postoperatively with the laparoscopic approach, and 4.3 \pm 1.0 days postoperatively with the robot-assisted approach. There were 19 surgical complications with the open approach $(57.6 \%)$, zero with the laparoscopic approach $(0.0 \%)$, and six with the robot-assisted approach $(42.9 \%)$. There were nine cases $(28.1 \%)$ of graft failure in open approach, zero cases $(0.0 \%)$ on the laparoscopic approach, and two cases $(14.3 \%)$ in the robot-assisted approach. Live birth after living-donor UTx was reported in 16 cases $(48.5 \%)$ with the open approach, zero cases $(0.0 \%)$ with the laparoscopic approach, and two cases (14.3\%) with the robot-assisted approach.

Clinical data for each operative approach with or without the uterine veins are also shown in Table 2. In the open approach, the mean operative time was 8 hours 45 minutes \pm 2 hours 39 minutes and the mean blood loss was $711 \pm 586 \mathrm{ml}$ in the cases where UVs were preserved $(\mathrm{n}=29)$; and in the cases where UVs were not preserved $(\mathrm{n}=4)$, the mean operative time was 6 hours 14 minutes \pm 0 hours 26 minutes, and the mean blood loss was $738 \pm 569 \mathrm{~mL}$. In the laparoscopic approach, the mean operative time was 4 hours 0 minutes \pm 0 hours 0 minutes and the mean blood loss was $100 \pm 0 \mathrm{~mL}$ in the UVs preserved cases $(\mathrm{n}=2)$, and the mean operative time was 3 hours 0 minutes \pm 0 hours 20 minutes and the mean blood loss was 100 $\pm 0 \mathrm{ml}$ in the non-UVs preserved cases $(\mathrm{n}=2)$. In the robot-assisted approach, the mean operative time 
and mean blood loss were 11 hours 19 minutes \pm 1 hour 8 minutes and $245 \pm 197 \mathrm{~mL}$ in the UVs preserved cases $(\mathrm{n}=12)$, respectively, and the mean operative time was 9 hours 1 minute \pm 3 hours 1 minute and the mean blood loss was $100 \pm 0 \mathrm{~mL}$ in the non-UVs preserved cases $(\mathrm{n}=2)$. In each approach, the operative time was reduced in the non-UVs preserved cases. The discharge time was $6.3 \pm 1.4$ postoperative days for the open approach in the UVs preserved cases, and $5.8 \pm 0.8$ days in the non-UVs preserved cases; $7.0 \pm$ 0.0 postoperative days for the laparoscopic approach in the UVs preserved cases, $6.0 \pm 0.0$ postoperative days in the non-UVs preserved cases. In the robot-assisted approach, the postoperative discharge time was $4.4 \pm 1.0$ days in the UVs preserved cases and $4.0 \pm 0.0$ days in the non-UVs preserved cases. There was little difference between patients with and without UVs preserved. Operative complications were found in 17 $(58.6 \%)$ cases for the open approach with UVs preserved, and in two $(50.0 \%)$ cases for non-UVs preserved. No complications were reported with the laparoscopic approach in both of the UVs preserved and non-UVs preserved cases. Complications tended to occur more frequently in the robot-assisted approach, with six cases $(50.0 \%)$ observed solely in the UVs preserved cases, with none in the non-UVs preserved cases. Complications were more frequent in the UVs preserved cases. In the robot-assisted approach, graft failure was reported in two patients $(16.7 \%)$ with UVs preserved. Live births after UTx utilising the laparoscopic approach were not reported in any of the papers included in this review. In the robot-assisted approach, one case (8.3\%) of a live birth was reported from the UVs preserved cases, and one (50.0\%) from the non-UVs preserved cases.

\section{Discussion}

Laparoscopic and robot-assisted approaches may contribute to less invasive living-donor surgery in UTx in the same way that they have led to less invasive surgery for other diseases. Similarly, the use of the OV or UOV for drainage, without UV, may also contribute to less invasive living-donor surgery.

Although UTx is an option for treatment of AUFI, there are still some issues to be solved: UTx is a non-vital organ transplantation and can be considered a transplantation to improve quality of life; despite the fact that UTx is such a medical treatment, surgery for living donors takes a long time and causes a large amount of blood loss, which places a heavy burden on the living donor.

One of the reasons for the high invasiveness of donor surgery is the difficulty in handling the complex venous system around the uterus. As the veins around the uterus make a network surrounding the ureter, they are difficult to isolate in the narrow operative field of the pelvic floor without sustaining damage. As a solution to this challenge, techniques in which the OV and/or UOV are used, instead of the UV, as drainage veins have been developed. In the UTx cases included in this review, only the UOV and OV were used, instead of the UV in each approach of living-donor surgery. With the exception of the open approach, the blood loss tended to be lower in non-UV preserved cases than in the UV preserved cases. This suggests that not using UV may contribute to a less invasive living-donor surgery for UTx.

On the other hand, there are concerns about the use of the UOV and OV as drainage veins. The first is whether these veins are sufficient for blood flow in the gestational uterus. Many pregnancies and live births have been reported after radical trachelectomy (RT), a fertility preservation treatment for cervical cancer in which the venous blood flow of the uterus is dependent on the UOV and OV, as well as UTx, using the UOV and OV only. ${ }^{30}$ Considering this fact, the venous flow required by the gestational uterus is preserved even if only the UOV and OV are used as drainage veins. In addition, considering that there have already been reports of live births in UTx without using the UV as a drainage vein, ${ }^{8,20}$ the use of the UOV and OV should be considered as an option for living-donor surgery. The second concern is the effect of vascular anastomosis. In UTx, donor vessels are mainly anastomosed to the recipient's external iliac vein, but it is unclear how the uterine enlargement caused by pregnancy affects the anastomosis sites and vessels. In particular, when the UOV is used, the effect of uterine augmentation during pregnancy is likely to be significant because of the short length of vessels that can be removed. It is hoped that more data about these points will be collected.

When the OV is used as a drainage vein, the impact of an enlarged gestational uterus may be lessened because a longer vessel can be removed than with UOV, but donor oophorectomy is unavoidable, and the associated complications must be considered. Bilateral oophorectomy in premenopausal patients results in 
the abolition of ovarian function and surgical primary ovarian insufficiency. Increased subjective symptoms, such as hot flashes and increased incidence of osteoporosis, cardiovascular disease, and non-alcoholic fatty liver, due to primary ovarian insufficiency have been reported. ${ }^{31}$ In addition, there have been some reports of an increase in all-cause mortality of about $28 \%$ in the absence of hormone replacement therapy for these patients. ${ }^{32,33}$ As UTx is performed to improve quality of life, complications in living donors should be avoided as much as possible, and a worsening living-donor prognosis is unacceptable. The use of the OV as a venous vessel on the premenopausal donor, where ovariectomy is inevitable, should be discussed.

Laparoscopic and robot-assisted approaches have advantages, such as improved magnification and small, minimally invasive wounds. These characteristics are being applied to clinical practice in UTx with the possibility of contributing to less invasive living-donor surgery. Previous reports comparing laparoscopic and open approaches for radical hysterectomy, in which the uterine artery and veins are handled as well as UTx living-donor surgery, have reported less intraoperative blood loss with laparoscopic approach than with open approach. ${ }^{34}$ In addition, robot-assisted approach has already been used in other organ transplant surgeries. ${ }^{35}$ It has already contributed to minimising invasive living-donor surgery and is, therefore, considered feasible. In this review, the laparoscopic and robot-assisted living-donor UTx operations also tended reduce blood loss compared to the open approach. There was also a tendency for patients to be discharged earlier with the robot-assisted approach than with the open approach. These findings suggest that the laparoscopic and robot-assisted approaches may contribute to less invasive UTx in the future.

On the other hand, the disadvantages and complications of laparoscopic and robot-assisted approaches need to be considered. Fourteen cases of robot-assisted surgeries were reported, but the average operative time was longer than that of the open approach. Temporary alopecia has been reported as a complication related to intraoperative positioning in robot-assisted approach. ${ }^{24}$ In addition, the main goal of UTx is to achieve live birth after transplantation. Although many live births have been reported for the open approach, partly because of the small number of procedures performed, only two live births have been reported for the laparoscopic and robot-assisted approach UTx. A long-term report on mental status after $\mathrm{UTx}^{17}$ found no clear results on how live birth affects mental status, but it is thought that live birth could affect the mental status of living donors. Although minimally invasive techniques for living donors are required, these need to be developed with the objectives of UTx in mind.

Open approach has a higher incidence of surgical complications and graft failure than minimally invasive approaches. However, the open approach has been performed since the inception of UTx, and surgical outcomes from that time are also included in this review. In addition, the robot-assisted approach is utilised in countries that perform more UTx by the open approach by surgeons who have sufficient experience with UTx surgery. The differences in clinical and operative outcomes between each approach may be influenced by these factors, which do not lead directly to the conclusion that the open approach is inferior to minimally invasive approaches. Further accumulation of data with regards the clinical outcomes of each approach is essential.

UTx from deceased donors has been clinically applied as a fundamental solution to the risks of living donors, and there are reports of live births after UTx from deceased donors. ${ }^{36,37}$ To investigate UTx from a deceased donor, basic research has also been conducted. ${ }^{38}$ However, this technique has challenges such as hormone replacement therapy for postmenopausal donors and difficulties in assessing the detailed uterine vasculature. The effects of these factors on graft implantation and live birth need to be examined. There are also many issues that need to be considered, such as what criteria are suitable for deceased donors for UTx and what surgical procedures for organ harvesting, such as hysterectomy, may be appropriate.

Limitations are present in this review. As this is a retrospective review, various confounding factors may be included, and it is not possible to say directly which approach is superior. Statistical comparisons with laparoscopic and robot-assisted approaches are particularly difficult to conduct because of the small number of procedures performed. In addition, this review only included original articles that met the inclusion criteria retrieved by PubMed. However, there are some articles that include unpublished data, such as live birth after laparoscopic UTx in India, and some press releases about the implementation of UTx in other countries..$^{5,39,40}$ 
If such information is included, the results may differ from those of this review.

This literature review on UTx living-donor surgical approaches, preserved veins, and operative and clinical outcomes was conducted with a focus on the laparoscopic approach and robot-assisted approach. Laparoscopic and robot-assisted approaches may contribute to less invasive living-donor surgery in UTx in the same way that they have led to less invasive surgery for other diseases. Similarly, the use of the OV or UOV as a drainage vein, without UV, may also contribute to less invasive living-donor surgery. However, there are few reports of live birth - the ultimate goal of UTx - with these new techniques. The application of these techniques needs to be thoroughly discussed.

\section{Acknowledgements}

None

\section{Disclosure of Interests}

All authors have no conflicts of interest to disclose regarding this article.

\section{Contribution to Authorship}

IK and KB conceived the idea for the review. YM and IK reviewed the literature and wrote the manuscript. DA reviewed and edited the manuscript.

\section{[Funding]}

No funding was used.

\section{References}

1. Herlin MK, Petersen MB, Brannstrom M. Mayer-Rokitansky-Kuster-Hauser (MRKH) syndrome: a comprehensive update. Orphanet J Rare Dis. 2020;15(1):214.

2. Hur C, Rehmer J, Flyckt R, Falcone T. Uterine Factor Infertility: A Clinical Review. Clin Obstet Gynecol. 2019;62(2):257-70.

3. Fageeh W, Raffa H, Jabbad H, Marzouki A. Transplantation of the human uterus. Int J Gynaecol Obstet. 2002;76(3):245-51.

4. Brännström M, Johannesson L, Bokström H, Kvarnström N, Mölne J, Dahm-Kähler P, et al. Livebirth after uterus transplantation. The Lancet. 2015;385(9968):607-16.

5. Daolio J, Palomba S, Paganelli S, Falbo A, Aguzzoli L. Uterine transplantation and IVF for congenital or acquired uterine factor infertility: A systematic review of safety and efficacy outcomes in the first 52 recipients. PLoS One. 2020;15(4):e0232323.

6. Brannstrom M, Johannesson L, Dahm-Kahler P, Enskog A, Molne J, Kvarnstrom N, et al. First clinical uterus transplantation trial: a six-month report. Fertil Steril. 2014;101(5):1228-36.

7. Chmel R, Novackova M, Janousek L, Matecha J, Pastor Z, Maluskova J, et al. Revaluation and lessons learned from the first 9 cases of a Czech uterus transplantation trial: Four deceased donor and 5 living donor uterus transplantations. Am J Transplant. 2019;19(3):855-64.

8. Testa G, McKenna GJ, Gunby RT, Jr., Anthony T, Koon EC, Warren AM, et al. First live birth after uterus transplantation in the United States. Am J Transplant. 2018;18(5):1270-4.

9. Puntambekar S, Telang M, Kulkarni P, Puntambekar S, Jadhav S, Panse M, et al. Laparoscopic-Assisted Uterus Retrieval From Live Organ Donors for Uterine Transplant: Our Experience of Two Patients. J Minim Invasive Gynecol. 2018;25(4):622-31. 
10. Wei L, Xue T, Tao KS, Zhang G, Zhao GY, Yu SQ, et al. Modified human uterus transplantation using ovarian veins for venous drainage: the first report of surgically successful robotic-assisted uterus procurement and follow-up for 12 months. Fertil Steril. 2017;108(2):346-56 e1.

11. Johannesson L, Kvarnstrom N, Molne J, Dahm-Kahler P, Enskog A, Diaz-Garcia C, et al. Uterus transplantation trial: 1-year outcome. Fertil Steril. 2015;103(1):199-204.

12. Brannstrom M, Bokstrom H, Dahm-Kahler P, Diaz-Garcia C, Ekberg J, Enskog A, et al. One uterus bridging three generations: first live birth after mother-to-daughter uterus transplantation. Fertil Steril. 2016;106(2):261-6.

13. Molne J, Broecker V, Ekberg J, Nilsson O, Dahm-Kahler P, Brannstrom M. Monitoring of Human Uterus Transplantation With Cervical Biopsies: A Provisional Scoring System for Rejection. Am J Transplant. 2017;17(6):1628-36.

14. Kvarnstrom N, Jarvholm S, Johannesson L, Dahm-Kahler P, Olausson M, Brannstrom M. Live Donors of the Initial Observational Study of Uterus Transplantation-Psychological and Medical Follow-Up Until 1 Year After Surgery in the 9 Cases. Transplantation. 2017;101(3):664-70.

15. Brannstrom M, Dahm-Kahler P, Kvarnstrom N, Akouri R, Rova K, Olausson M, et al. Live birth after robotic-assisted live donor uterus transplantation. Acta Obstet Gynecol Scand. 2020.

16. Brannstrom M, Dahm-Kahler P, Ekberg J, Akouri R, Groth K, Enskog A, et al. Outcome of Recipient Surgery and 6-Month Follow-Up of the Swedish Live Donor Robotic Uterus Transplantation Trial. J Clin Med. 2020;9(8).

17. Jarvholm S, Dahm-Kahler P, Kvarnstrom N, Brannstrom M. Psychosocial outcomes of uterine transplant recipients and partners up to 3 years after transplantation: results from the Swedish trial. Fertil Steril. 2020;114(2):407-15.

18. Brannstrom M, Kvarnstrom N, Groth K, Akouri R, Wiman L, Enskog A, et al. Evolution of surgical steps in robotics-assisted donor surgery for uterus transplantation: results of the eight cases in the Swedish trial. Fertil Steril. 2020.

19. Broecker V, Brannstrom M, Ekberg J, Dahm-Kahler P, Molne J. Uterus transplantation: Histological findings in explants at elective hysterectomy. Am J Transplant. 2020.

20. Huang Y, Ding X, Chen B, Zhang G, Li A, Hua W, et al. Report of the first live birth after uterus transplantation in People's Republic of China. Fertil Steril. 2020.

21. Testa G, Koon EC, Johannesson L, McKenna GJ, Anthony T, Klintmalm GB, et al. Living Donor Uterus Transplantation: A Single Center's Observations and Lessons Learned From Early Setbacks to Technical Success. Am J Transplant. 2017;17(11):2901-10.

22. Ramani A, Testa G, Ghouri Y, Koon EC, Di Salvo M, McKenna GJ, et al. DUETS (Dallas UtErus Transplant Study): Complete report of 6-month and initial 2-year outcomes following open donor hysterectomy. Clin Transplant. 2020;34(1):e13757.

23. Johannesson L, Koon EC, Bayer J, McKenna GJ, Wall A, Fernandez H, et al. DUETS (Dallas UtErus Transplant Study): Early Outcomes and Complications of Robot-Assisted Hysterectomy for Living Uterus Donors. Transplantation. 2020.

24. Testa G, McKenna GJ, Bayer J, Wall A, Fernandez H, Martinez E, et al. The Evolution of Transplantation From Saving Lives to Fertility Treatment: DUETS (Dallas UtErus Transplant Study). Ann Surg. 2020.

25. Chmel R, Cekal M, Pastor Z, Chmel R, Jr., Paulasova P, Havlovicova M, et al. Assisted Reproductive Techniques and Pregnancy Results in Women with Mayer-Rokitansky-Kuster-Hauser Syndrome Undergoing Uterus Transplantation: the Czech Experience. J Pediatr Adolesc Gynecol. 2020. 
26. Chmel R, Novackova M, Pastor Z. Lessons learned from the Czech uterus transplant trial related to surgical technique that may affect reproductive success. Aust N Z J Obstet Gynaecol. 2020;60(4):625-7.

27. Brucker SY, Brannstrom M, Taran FA, Nadalin S, Konigsrainer A, Rall K, et al. Selecting living donors for uterus transplantation: lessons learned from two transplantations resulting in menstrual functionality and another attempt, aborted after organ retrieval. Arch Gynecol Obstet. 2018;297(3):675-84.

28. Brucker SY, Strowitzki T, Taran FA, Rall K, Scholler D, Hoopmann M, et al. Living-Donor Uterus Transplantation: Pre-, Intra-, and Postoperative Parameters Relevant to Surgical Success, Pregnancy, and Obstetrics with Live Births. J Clin Med. 2020;9(8).

29. Puntambekar S, Puntambekar S, Telang M, Kulkarni P, Date S, Panse M, et al. Novel Anastomotic Technique for Uterine Transplant Using Utero-ovarian Veins for Venous Drainage and Internal Iliac Arteries for Perfusion in Two Laparoscopically Harvested Uteri. J Minim Invasive Gynecol. 2019;26(4):628-35.

30. Tamauchi S, Kajiyama H, Sakata J, Sekiya R, Suzuki S, Mizuno M, et al. Oncologic and obstetric outcomes of early stage cervical cancer with abdominal radical trachelectomy: Single-institution experience. J Obstet Gynaecol Res. 2016;42(12):1796-801.

31. Matsuo K, Gualtieri MR, Cahoon SS, Jung CE, Paulson RJ, Shoupe D, et al. Surgical menopause and increased risk of nonalcoholic fatty liver disease in endometrial cancer. Menopause. 2016;23(2):189-96.

32. Zurfluh S, Nickler M, Ottiger M, Steuer C, Kutz A, Christ-Crain M, et al. Dihydrotestosterone is a predictor for mortality in males with community-acquired pneumonia: results of a 6-year follow-up study. Respir Res. 2018;19(1):240.

33. Faubion SS, Kuhle CL, Shuster LT, Rocca WA. Long-term health consequences of premature or early menopause and considerations for management. Climacteric. 2015;18(4):483-91.

34. Tantitamit T, Huang KG, Lee CL. Laparoscopic versus open radical hysterectomy in women with early stage cervical cancer: A systematic review and meta-analysis. Taiwan J Obstet Gynecol. 2020;59(4):481-8.

35. Giacomoni A, Di Sandro S, Lauterio A, Concone G, Buscemi V, Rossetti O, et al. Robotic nephrectomy for living donation: surgical technique and literature systematic review. Am J Surg. 2016;211(6):1135-42.

36. Ejzenberg D, Andraus W, Baratelli Carelli Mendes LR, Ducatti L, Song A, Tanigawa R, et al. Livebirth after uterus transplantation from a deceased donor in a recipient with uterine infertility. Lancet. 2019;392(10165):2697-704.

37. Flyckt R, Falcone T, Quintini C, Perni U, Eghtesad B, Richards EG, et al. First birth from a deceased donor uterus in the United States: from severe graft rejection to successful cesarean delivery. Am J Obstet Gynecol. 2020;223(2):143-51.

38. Kisu I, Umene K, Adachi M, Emoto K, Nogami Y, Banno K, et al. Allowable warm ischemic time and morphological and biochemical changes in uterine ischemia/reperfusion injury in cynomolgus macaque: a basic study for uterus transplantation. Hum Reprod. 2017;32(10):2026-35.

39. Zaami S, Di Luca A, Marinelli E. Advancements in uterus transplant: new scenarios and future implications. Eur Rev Med Pharmacol Sci. 2019;23(2):892-902.

40. Kisu I, Liu Y, Chen G, Song MJ, Chang CY, Koon TH, et al. Current Progress in Uterus Transplantation Research in Asia. J Clin Med. 2019;8(2).

\section{Tables and Figures}

Figure 1 . Drainage vein options for uterus transplantation.

In the gold standard of uterine transplantation, the uterine veins from the internal iliac vein are used as drainage veins. However, this surgery is challenging because these vessels are located in the deep pelvic floor and surround the ureter. To minimise the invasiveness of living-donor surgery, the use of the ovarian vein 
or the utero-ovarian vein - which runs continuously from the ovarian vein through the mesosalpinx - as the drainage vein, has been considered as an alternative to the use of the uterine vein. Ut, uterus; UV, uterine vein; UOV, utero-ovarian vein; OV, ovarian vein

Figure 2 . Flowchart of article selection.

On 13 October, 2020, an article search was conducted on PubMed according to the search strategy. Of 2382 articles, 26 original articles were finally included in the review. They include the operative and clinical outcome data of the UTx living donor. UTx, uterine transplantation

Table 1 . Reported operative and clinical data of living-donor surgery for uterus transplantation

Table 2 . Operative and clinical data for each operative approach with or without using the uterine vein

\section{Hosted file}

Table_1.pdf available at https://authorea.com/users/375649/articles/492812-operative-andclinical-outcomes-of-minimally-invasive-living-donor-surgery-on-uterus-transplantationa-literature-review

\section{Hosted file}

Table_2.pdf available at https://authorea.com/users/375649/articles/492812-operative-andclinical-outcomes-of-minimally-invasive-living-donor-surgery-on-uterus-transplantationa-literature-review

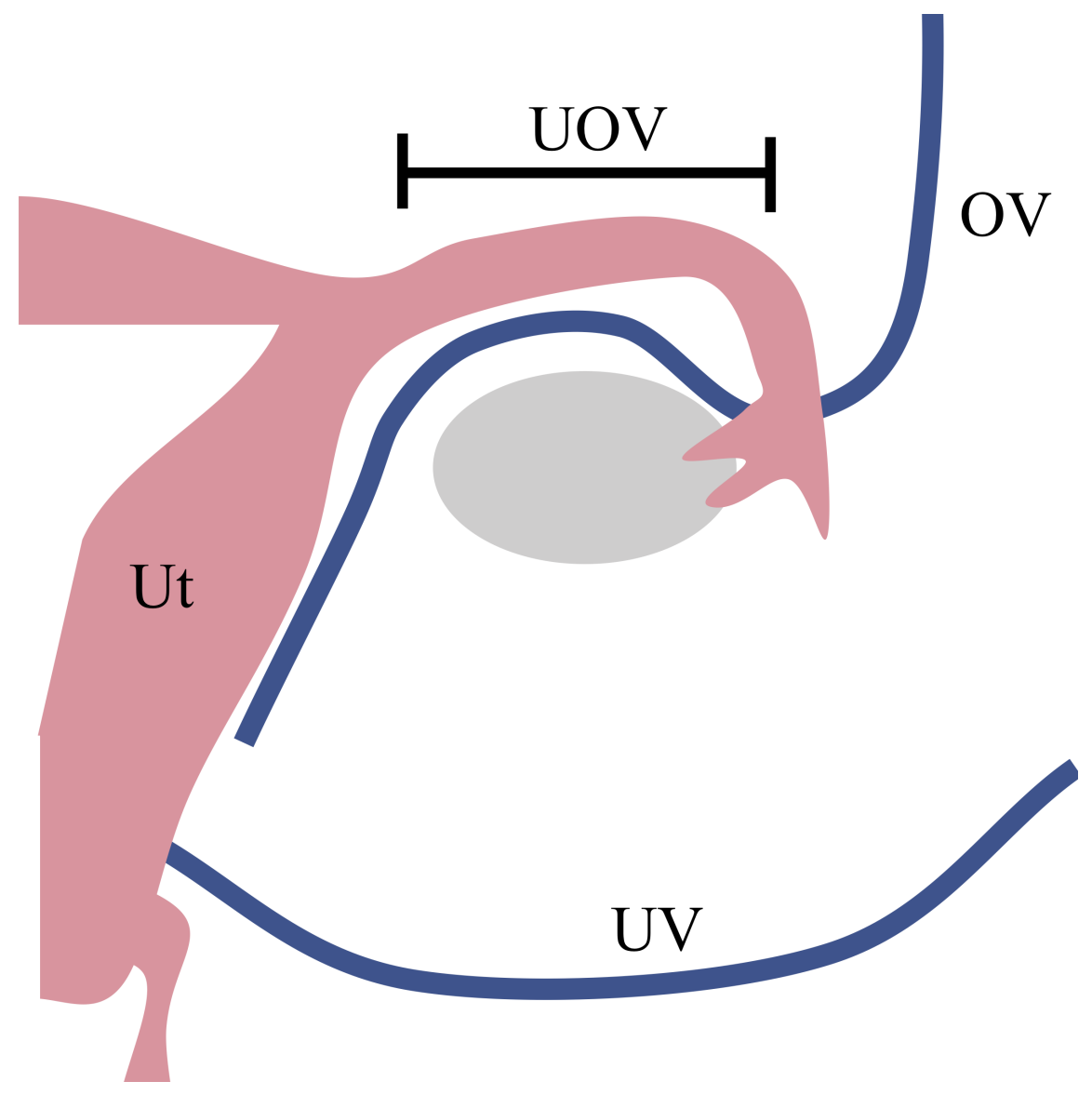


Articles identified

through the searching strategy

$$
(\mathrm{n}=2382)
$$

Articles excluded because of written

in non-English language

$(n=294)$

\begin{tabular}{|c|l|l|}
\hline \multicolumn{1}{|c|}{$\begin{array}{c}\text { Articles written in English } \\
(\mathrm{n}=2088)\end{array}$} & $\begin{array}{l}\text { Articles excluded because of } \\
\text { - not about UTx } \\
\text { - on animal model } \\
\text { - not original article } \\
(\mathrm{n}=1914)\end{array}$ \\
\hline $\begin{array}{c}\text { Articles considered eligible after the } \\
\text { examination of the full text } \\
(\mathrm{n}=174)\end{array}$ & $\begin{array}{l}\text { Articles excluded because there are } \\
\text { no information about operative and } \\
\text { clinical outcome about living donor. } \\
\text { (n=148) }\end{array}$ \\
\hline $\begin{array}{c}\text { Articles included in the final analysis } \\
(\mathrm{n}=26)\end{array}$ &
\end{tabular}

